\title{
Microdomain Organization of the Plasma Membrane in Keratinocytes
}

\author{
Dina Vind-Kezunovic and Robert Gniadecki*
}

Department of Dermatology, University of Copenhagen, Bispebjerg Bakke 23, 2400 Copenhagen NV, Denmark

\begin{abstract}
Plasma membrane of the keratinocyte is organized in a domain structure comprising the nanoscale areas of the lipid in the liquid ordered state (comparable to lipid rafts) surrounded by the liquid-disordered lipid fraction. In this review we summarize the current state of knowledge on the microdomain organization of the keratinocytes plasma membrane. Application of the fluorescent derivative of cholera toxin B1 subunit cross-links these domains into larger, nanometerscale units in which molecular movement of proteins and lipids is retarded. Microdomain cross-linking is particularly effective in the basal portion of the cell within the focal junctions. Thus, junctional structures can behave as molecular sieves in which the density of microdomains is increased. Since lipid rafts play a role in transmembrane signal transduction (see other articles in this supplementum) these finding can explain the compartmentalization of signaling processes in the membrane. Research has also revealed how the microdomain organization of keratinocytes plasma membrane determines membrane curvature and regulates the formation of exocytotic vesicles and filopodia-like structures. Coalescence of a large number of microdomains into larger subunits can bend the membrane by the force generated on he lipid ordered/lipid disordered interphase. Microdomain composition of the membrane can also add another dimension to the explanation of skin pathology such as epidermal hyperplasia, apoptosis, cell-cell adhesion and disorders of cornification.
\end{abstract}

\section{ROLE OF CHOLESTEROL IN THE MAINTENANCE OF THE PLASMA MEMBRANE STRUCTURE}

The fluid mosaic model of the plasma membrane proposed by Singer and Nicolson in the early 1970s provided the basic understanding of the plasma membrane structure. In a double layer of fluid lipids, the proteins diffuse without restriction in the plane of the plasma membrane [1]. However, the experimental data of the last decade have indicated that the plasma membrane is a much more complex structure characterized by lateral inhomogeities [2-4]. Firstly, the plasma membrane has an asymmetric leaflet organization. In the outer exoplasmic leaflet, the composition of lipids comprises mainly sphingomyelin and phosphatidylcholine, whereas the inner cytoplasmic leaflet is rich in amines (phosphatidylethanolamine, phosphatidylserine) [5]. It has also become apparent that lipids form nanometre-sized assemblies and that transmembrane proteins further subdivide the plasma membrane into distinct compartments influencing the lateral movement [4, 6, 7]. The domain structure of the plasma membrane is a controversial issue, and because of its importance for the understanding of basic cellular processes such as signal transduction and transmembrane transport, it is a subject of intensive interdisciplinary research [8].

Cholesterol is an essential plasma membrane lipid, accounting for 20-25\% of the plasma membrane lipid content. Cholesterol organizes lipid structure [9, 10]. Studies on lipid monolayers have shown that cholesterol prevents the crystallization of fatty acyl chains by inserting between

*Address correspondence to this author at the Department of Dermatology, University of Copenhagen, Bispebjerg Hospital, Bispebjerg Bakke 23, 2400 Copenhagen NV, Denmark; Tel: (+45) 35313165; Fax: (+45) 35315050;

E-mail:rg01@bbh.regionh.dk them, and it has a condensing effect because it decreases the surface area of saturated lipids [5]. Because sphingolipids have long saturated hydrocarbon chains, they easily intercalate cholesterol between the tails. Sphingolipids and cholesterol therefore associate with each other, creating, together with saturated phospholipids, a higher molecular order termed the "liquid-ordered" $\left(\mathrm{L}_{\mathrm{o}}\right)$ phase (Fig. 1A). In contrast, the bulk membrane unsaturated phospholipids with kinked hydrocarbon chains are more loosely organized in the "liquid-disordered" $\left(\mathrm{L}_{\mathrm{d}}\right)$ phase as shown in Fig. (1B). In a membrane without or with a relatively small amount of cholesterol, a third phase exists which is composed of saturated lipids with a high order of acyl chains organized similarly to $\mathrm{L}_{0}$ phase lipids. This phase is termed as the gel phase (Fig. 1C). Gel phase lipids diffuse with very low transitional freedom (lateral mobility in the plane of the membrane) compared to the unsaturated $\mathrm{L}_{\mathrm{d}}$ lipids $[11,12]$, and they do not exist in mammalian cells [10]. $\mathrm{L}_{\mathrm{o}}$ phase lipids diffuse with a lateral mobility similar to that of $\mathrm{L}_{\mathrm{d}}$ lipids, but because of the higher order, the diffusion is two to three fold slower than in the $L_{d}$ phase $[11,12]$.

One of the most debated models of plasma membrane organization is the hypothesis of "lipid rafts", which proposes that cholesterol, sphingomyelin and saturated glycolipids assemble into distinct domains [8, 13]. This concept helps to explain the mechanisms of lipid and protein trafficking to and from the apical cell membrane and signal transduction [14]. Moreover, the raft concept has been useful in the explanation of some pathological conditions such as Alzheimer's disease, atherosclerosis, bacterial and viral infections, cancer and autoimmune disease [15].

The concept of lipid rafts was proposed in the late eighties by Simons and Van Meer to explain why glycosphingolipids and glycoproteins were delivered to the exoplasmic leaflet of the apical but not the basolateral 
A

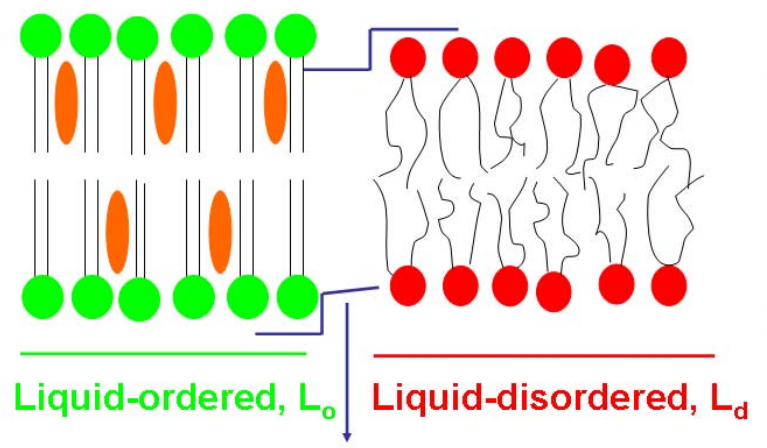

C
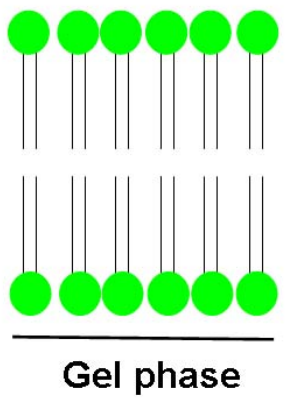

\section{Line tension}

Fig. (1). Lipid organization in the plasma membrane. Organization of (A) the liquid-ordered phase (orange ovals represent cholesterol). Cholesterol is inserted perpendicular to the two plasma membrane leaflets and acts as a spacer between especially long-tailed saturated glycosphingolipids. The polar moiety of cholesterol is the hydroxyl group $\mathrm{OH}$ which interacts with the polar groups of the saturated lipids, whereas the 4-ring steroid backbone with the hydrophobic tail is embedded in the hydrophobic centre of the bilayer [10]. (B) the liquiddisordered phase and $(\mathbf{C})$ the Gel phase. The boundary hydrophobic mismatch between the $\mathrm{L}_{\mathrm{o}}$ and $\mathrm{L}_{\mathrm{d}}$ domains gives rise to the line tension at domain boundary edge (blue zigzag line)

membrane in polarized cells $[14,16]$. The belief in the existence of lipid raft-like domains in living cells was reinforced by the experimental isolation of membranes with lipid and protein compositions identical to those of the apical Madin-Darby canine kidney (MDCK) membrane after treatment with the non-ionic detergent Triton $\mathrm{X}-100$ at $0^{\circ} \mathrm{C}$ [17]. Brown and Rose showed in 1992 that after sucrose detergent centrifugation, sphingolipid and cholesterol were localized in the light (5\%-30\%) fractions together with glycosylphosphatidyl inositol (GPI)-anchored proteins, which are other apical membrane components [17]. Numerous studies correlated the light membrane fractions with lipid rafts and showed that some proteins, including those involved in signal transduction, are reproducibly recovered from these fractions. Raft-associated proteins can be transmembrane proteins and be coupled to rafts by doubly acylated proteins like Src tyrosine kinases, or through myristoylation or palmitoylation to the lipids $[18,19]$.

Examples of these putative raft-associated proteins include growth factor receptors (e.g. the epidermal growth factor receptor (EGFR)), T cell receptor, B cell receptor, immunoglobulin $\mathrm{E}$ receptor, Src-family proteins and integrins $[18,19]$.

How raft-precursor lipids and proteins are sorted from the trans-Golgi network to be organized in raft domains at the cell surface has been explained by Anderson and Jacobson, who proposed that so-called lipid "shells" were the missing link in the biogenesis of rafts. This model suggests that membrane trafficking is facilitated by the formation of assemblies, each consisting of a single protein surrounded by around 80 molecules of cholesterol and sphingolipids forming a shell-like structure with the size of approximately $7 \mathrm{~nm}[20]$.

Because of their submicroscopic size, the lipid rafts have been notoriously difficult to image. Using advanced microscopic techniques like fluorescence depolarization, Pralle et al. documented that raft-associated GPI-proteins float as diffusional entities in the plasma membrane with a size of approximately $50 \mathrm{~nm}$ on a time scale of minutes [21]. Varma et al. showed by fluorescence resonance energy transfer (FRET) that clusters of raft-associated folate receptors in fibroblasts were below $70 \mathrm{~nm}$ in diameter [22]. By single dye tracking, Schutz et al. demonstrated the presence of approximately $0.7 \mu \mathrm{m}$ raft domains at room temperature (RT) in human coronary artery smooth muscle cells [23]. Importantly, Eggeling et al. recently observed nanoscale dynamics of membrane sphingolipids and a GPI protein anchor in living PtK2 cells by the technique of stimulated emission depletion far-field fluorescence nanoscopy at physiological conditions. In contrast to the phosphoroglycerolipid phosphatidylethanolamine these raft markers are entrapped within cholesterol-enriched complexes below $20 \mathrm{~nm}$ size at a time-scale of 10-20 ms [24].

Given the difficulties in studying the rafts in living cells, much of the current understanding of lipid domain formation in living cells also originates from studies on artificial model membranes [25]. Lipid raft-like domains in living cells have been equated to the $\mathrm{L}_{\mathrm{o}}$ phase in model membranes. Model membrane mixtures of equal amounts of cholesterol $/ \mathrm{L}_{\mathrm{o}} / \mathrm{L}_{\mathrm{d}}$ lipid are studied because they mimic the exoplasmic leaflet lipid content of the MDCK plasma membrane. Lipid phase properties are influenced by factors such as temperature, $\mathrm{pH}$, ionic strength and pressure [25]. The ordered raft-lipids are characterized by having a higher melting temperature $\left(\mathrm{T}_{\mathrm{m}}\right)$ compared to disordered $\mathrm{L}_{\mathrm{d}}$ lipids. When lipid phases are visualized by fluorescence-labeled lipid probes with affinity of either of the lipid phases using high technology microscopy, a phase diagram can be constructed [11]. Because saturated lipid acyl chains of $\mathrm{L}_{\mathrm{o}}$ lipids are stretched and elongated in comparison with the kinked unsaturated acyl tails of $\mathrm{L}_{\mathrm{d}}$ lipid phase, the bilayer differs in thickness at the phase boundary as shown in Fig. (1). The exposure of the hydrophobic parts of $\mathrm{L}_{\mathrm{o}}$ lipids causes a hydrophobic mismatch, which consequently causes a line tension to arise 
at the boundary edge of the $\mathrm{L}_{\mathrm{o}}$ and $\mathrm{L}_{\mathrm{d}}$ lipid phases. This energy is minimized when $L_{o}$ and $L_{d}$ lipids segregate into distinct domains [26, 27]. Model membranes are, however, much simpler than plasma membranes because they are composed of few different lipid species symmetrically organized in the two lipid leaflets [3, 28].

Even more importantly, artificial membranes do not include the proteins, which are important players in the plasma membrane organization according to many studies $[3,4,6]$. Gaus et al. showed that both transmembrane proteins attached to the cytoskeleton in $\mathrm{T}$ lymphocytes, and integrin-enriched focal adhesions in endothelial cells regulate the lipid domain organization [29,30]. Lillemeier et $a l$. have shown by transmission electron microscopy (TEM) that the plasma membrane is subdivided into distinct cholesterol and protein-rich zones, probably by membrane integral proteins attached to the underlying cytoskeleton. These "islands" consist of both raft and non-raft protein clusters. The islands are surrounded by a protein-free and cholesterol-poor membrane [7]. Kusumi and co-workers documented that transmembrane proteins as pickets interact with the actin cytoskeleton, organized in a fence-like structure which compartmentalizes the plasma membrane into nanometre-sized zones (ranging from 30-230 nm depending on the cell type used) [4]. The molecular diffusion within these compartments is Brownian random, whereas movement between the compartments occurs as long-range hop-diffusion. The picket-and-fence structures slow molecular movement in the plasma membrane by a factor 5 50 compared to what has been observed in model membranes [4].

The understanding of the lateral mobility of the molecules in the plasma membrane is essential for the explanation of cellular signaling. When membraneembedded receptors are brought into proximity to other receptors, cell signaling is facilitated. This explains why molecular diffusion has a high influence on the cellular signal transduction. Moreover, the segregation of receptors into distinct lipid phases is also believed to influence signal transduction [18]. For example, in the case of tyrosine kinases, their association with raft lipids protects against the phosphatases which often tend to be associated with the nonraft membrane compartment [18].

\section{ROLES OF CHOLESTEROL AND MICRODOMAINS IN THE PLASMA MEMBRANE OF KERATINO- CYTES}

The skin protects the body against mechanical and chemical damage, solar ultraviolet radiation and the intrusion of the microorganisms. The skin barrier controls the outward diffusion of water and electrolytes and prevents desiccation. These properties are due to the unique structure of the stratum corneum. Its essential component is a hydrophobic lipid matrix consisting of lamellar lipid membranes [31]. The lipid mass of the stratum corneum is $25 \%$ cholesterol, $50 \%$ ceramide and $15 \%$ free fatty acids, with only a low concentration of phospholipids [31].

Cholesterol is a major regulator in the formation of the cornified envelope, which is a layer of cross-linked protein deposited under the plasma membrane [31]. Cholesterol is produced in the skin from endogenous synthesis via the mevalonate pathway with 3-hydroxy-3-methylglutaryl coenzyme A (HMG-CoA) reductase being the rate-limiting enzyme. Cholesterol can also be delivered from exogenous sources. Under physiological circumstances when the lipid barrier is disrupted, the activity of HMG-CoA reductase is enhanced [31]. The enzyme activity can be inhibited by topical application of statins, which causes a delayed recovery of the permeability barrier function in terms of both lamellar body structure and deposition, with consequent abnormalities in the lamellar membranes in the lower stratum corneum [32].

Our laboratory has previously shown that in the normal epidermis, raft marker glycolipid and ganglioside $\mathrm{GM}_{1}$ can be visualized with cholera toxin $\mathrm{B}$ subunit-fluorescein isothiocyanate (CTB-FITC). $\mathrm{GM}_{1}$ is enriched in transient amplifying keratinocytes localized in the rete ridges of the basal layer [33]. In skin biopsies from patients with hidradenitis suppurativa, the cells were enriched in both $\mathrm{GM}_{1}$ and $\beta 1$ integrin CD29 [34] in the sinus tracts. Because integrins in keratinocytes are important in cell movement and they are expressed in benign hyperproliferative processes [35], it was proposed that the occurrence of $\mathrm{GM}_{1}$ and CD29 enriched raft-like domains in this particular cell type plays a role in epidermal migration [34].

The lipid microdomain organization of the keratinocyte plasma membrane has not been studied in depth, but preliminary published data suggest that lipid raft-like domains are found in keratinocytes because $\mathrm{CTB}^{\text {bright }}$ domains are visualized in normal human keratinocytes (NHK) [33] and in immortalized HaCaT keratinocytes [36]. Unattached $\mathrm{HaCaT}$ keratinocytes in suspension present with a homogeneous staining of the plasma membrane by CTBFITC. In contrast, 24 hours later, when cells attached to the coverslips and reached confluence, discernable domains of $\mathrm{GM}_{1}$ distributed into junction-like structures at the basal membrane portion, suggesting a preference for cellular contacts and focal adhesions [36].

We hypothesized that the $\mathrm{CTB}^{\text {bright }}$ domains in the plasma membrane of $\mathrm{HaCaT}$ cells represented aggregates of lipid rafts mainly on the basis of their enrichment in $\mathrm{GM}_{1}$ gangliosides and cholesterol [37] and because CTB colocalized with raft markers flotillin 1 and 2, caveolin 1 and 2 [38] and the epidermal growth factor receptor (EGFR) [39] in double-labeling experiments.

This assumption was in line with the current concept of lipid rafts, which suggests that these microdomains are dynamic 10-200 nm domains which can coalesce into larger $(>200 \mathrm{~nm}$ ) domains stabilized by lipid-lipid, lipid-protein or protein-protein interactions [13]. Thus the observed $\mathrm{CTB}^{\text {bright }}$ domains could have represented aggregates of nanoscale rafts, similar to the superraft-like structures in the enterocyte brush border or the immunological synapse [40, 41].

Unexpectedly, however, $\mathrm{L}_{\mathrm{d}}$ markers Alexa Fluor (AF)conjugated transferrin, which labels the transferrin receptor, and the lipophilic dye 1,1'-dioctadecyl-3,3,3',3'tetramethylindocarbocyanine perchlorate $\left(\right.$ DiI- $\left._{18: 0}\right)$ were also enriched within the $\mathrm{CTB}^{\text {bright }}$ domains [38]. FRAP experiments further showed that the lateral mobilities of CTB-FITC, EGFR, AF-transferrin and DiI-C $\mathrm{C}_{18: 0}$ were 

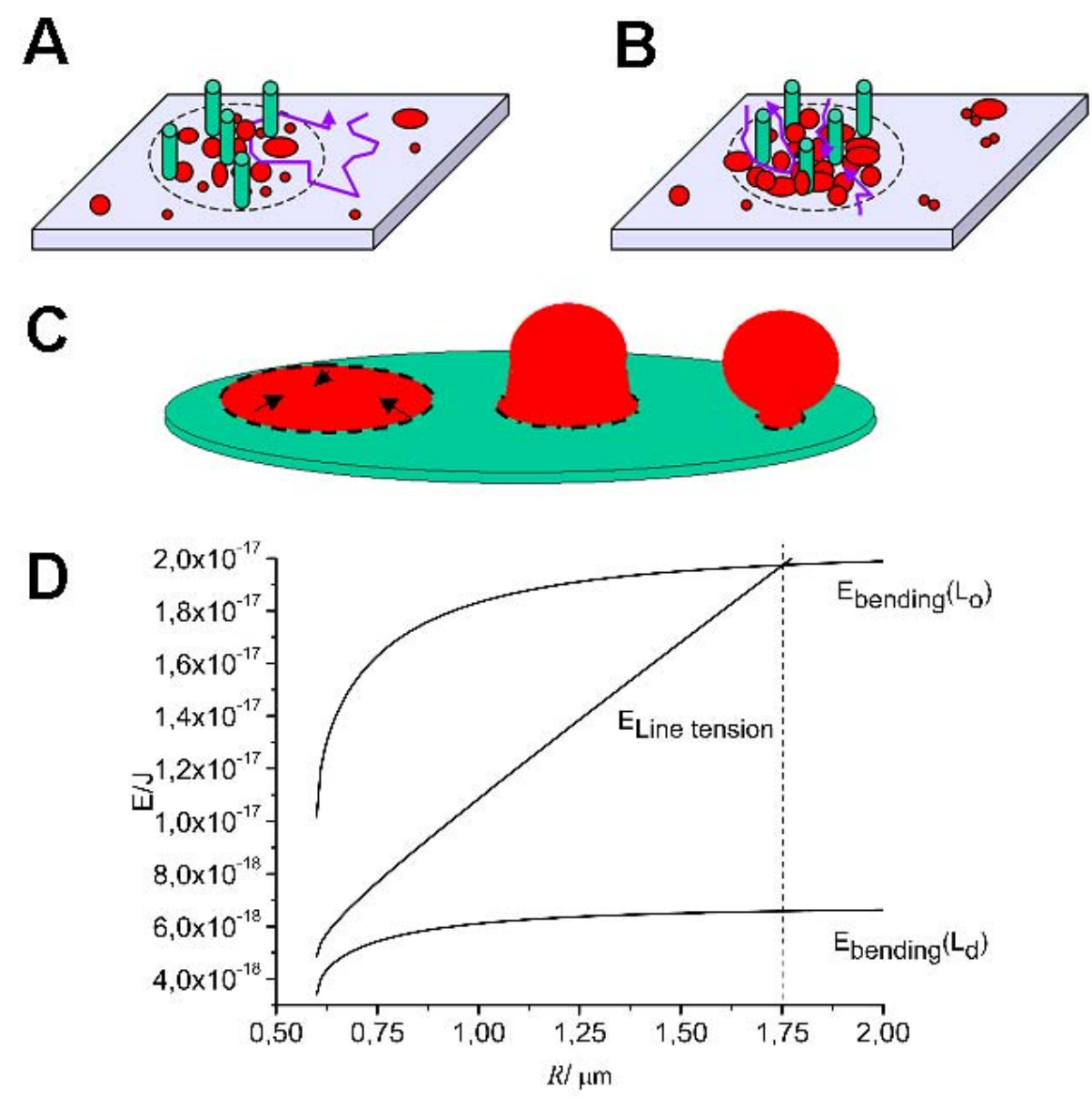

Fig. (2). (A) Focal junctions contain high concentrations of lipid raft-like domains (red ovals), probably via their entrapment by arrays of transmembrane proteins (green cylinders). These domains form meshwork-like structures that retard the motion (purple arrows) of the $\mathrm{L}_{\mathrm{d}}$ like phase (blue). (B) These effects are enhanced by $\mathrm{L}_{\mathrm{o}}$ domain cross-linking by CTB or domain coalescence in cholesterol-depleted cells in which the $\mathrm{L}_{\mathrm{d}}$-like phase becomes enmeshed within the dense labyrinth of $\mathrm{L}_{\mathrm{o}}$ domains. $(\mathbf{C})$ The Line tension energy ( $\mathrm{E}_{\mathrm{Line}}$ tension ) can be reduced if the plasma membrane bulges out of the plane to form vesicles, thereby reducing the domain edge (broken line). (D) Plots of Energy/J vs radius of the calculated vesicle radius $(R)$. Curves show $\mathrm{E}_{\text {Bending }}\left(\mathrm{L}_{\mathrm{d}}\right), \mathrm{E}_{\mathrm{Bending}}\left(\mathrm{L}_{\mathrm{o}}\right)$, and $\mathrm{E}_{\text {Line tension }}$ as function of $R$. Note that $\left(\mathrm{E}_{\mathrm{Line}}\right.$ tension $>\mathrm{E}_{\text {Bending }}\left(\mathrm{L}_{\mathrm{d}}\right)$ for all values of $R$.

diminished in $\mathrm{CTB}^{\text {bright }}$ domains and further reduced after cholesterol depletion by $\mathrm{M} \beta \mathrm{CD}[38,39]$. Since the $\mathrm{CTB}^{\text {bright }}$ domains were mostly visible on the basal portion of the membrane where cells adhere to the glass, we speculated that they represented one of the adhesive structures. Indeed, an adhesion protein $\alpha 4 \beta 1$ integrin spatially overlapped with the CTB-FITC and DiI-C $18: 0$, suggesting that CTB $^{\text {bright }} \mathrm{L}_{0}$-like domains represented focal junctions. This led us to the development of a model of focal junctions as molecular sieves restricting the lateral mobility of the molecules which happen to migrate through them as shown in Figs. (2A, B) and [38].

In the apical $\mathrm{HaCaT}$ keratinocyte plasma membrane stained with CTB-FITC and DiI-C $\mathrm{C}_{18: 0}$, we observed spherical 1-2 micrometre budding vesicles, which were predominantly enriched in DiI-C $18: 0$, whereas $\mathrm{GM}_{1}$ was weakly stained [42]. Cholesterol depletion with $\mathrm{M} \beta \mathrm{CD}$ enhanced the vesiculation of DiI- $\mathrm{C}_{18: 0}$ and other selected DiI $\mathrm{L}_{\mathrm{d}}$ markers, including short-tailed $\quad 1,1^{\prime}$-didodecyl-3,3,3',3' -tetramethylindocarbo- cyanine perchlorate and di-unsaturated 1,1'-dilinoleyl-3,3, $3^{\prime}, 3^{\prime}$-tetramethylindocarbocyanine perchlorate, suggesting that cholesterol plays a role in the control of exocytic vesiculation. It was conceivable that the line tension arising at the edge of the $\mathrm{L}_{\mathrm{o}} / \mathrm{L}_{\mathrm{d}}$ boundaries could provide a force strong enough to cause membrane bending and vesicle formation as shown in Fig. (2C). An analysis of the energetic requirements for vesiculation confirmed this hypothesis and revealed that a coalescence of smaller $\mathrm{L}_{\mathrm{d}}$-like domains at a micrometre scale makes it energetically more favourable for the vesicles to be composed of $\mathrm{L}_{\mathrm{d}}$-like lipids than of $\mathrm{L}_{0}$-like lipids (Fig. 2D and [42]).

Taken together, these studies shed new light on the lipid domain organization of keratinocyte plasma membrane and point to the potential importance of the cholesterol and membrane microdomains in the regulation of diverse processes such as membrane receptor signaling, cell adhesion and vesicle formation. Membrane cholesterol can 
provide an interesting target for pharmacological manipulation of keratinocyte metabolism.

\section{REFERENCES}

[1] Singer SJ, Nicolson GL. Fluid Mosaic Model of Structure of CellMembranes. Science 1972; 175: 720-31.

[2] van Meer G. Cellular lipidomics. EMBO J 2005; 24: 3159-65.

[3] Feigenson GW. Phase boundaries and biological membranes. Annu Rev Biophys Biomol Struct 2007; 36: 63-77.

[4] Kusumi A, Nakada C, Ritchie K, et al. Paradigm shift of the plasma membrane concept from the two-dimensional continuum fluid to the partitioned fluid: high-speed single-molecule tracking of membrane molecules. Annu Rev Biophys Biomol Struct 2005; 34: 351-78.

[5] Burger K, Gimpl G, Fahrenholz F. Regulation of receptor function by cholesterol. Cell Mol Life Sci 2000; 57: 1577-92.

[6] Silvius J. Lipid microdomains in model and biological membranes: how strong are the connections?. Q Rev Biophys 2005; 38: 373-83.

[7] Lillemeier BF, Pfeiffer JR, Surviladze Z, Wilson BS, Davis MM. Plasma membrane-associated proteins are clustered into islands attached to the cytoskeleton. Proc Natl Acad Sci USA 2006; 103: 18992-97.

[8] Jacobson K, Mouritsen OG, Anderson RG. Lipid rafts: at a crossroad between cell biology and physics. Nat Cell Biol 2007; 9: 7-14.

[9] Ikonen E. Cellular cholesterol trafficking and compartmentalization. Nat Rev Mol Cell Biol 2008; 9: 125-38.

[10] Maxfield FR, Tabas I. Role of cholesterol and lipid organization in disease. Nature 2005; 438: 612-21.

[11] Hancock JF. Lipid rafts: contentious only from simplistic standpoints. Nat Rev Mol Cell Biol 2006; 7: 456-62.

[12] Simons K, Vaz WL. Model systems, lipid rafts, and cell membranes. Annu Rev Biophys Biomol Struct 2004; 33: 269-95.

[13] Pike LJ. Rafts defined: a report on the Keystone Symposium on Lipid Rafts and Cell Function. J Lipid Res 2006; 47: 1597-98.

[14] Simons K, Ikonen E. Functional rafts in cell membranes. Nature 1997; 387: 569-72.

[15] Simons K, Ehehalt R. Cholesterol, lipid rafts, and disease. J Clin Invest 2002; 110: 597-03.

[16] Simons K, van Meer G. Lipid sorting in epithelial cells. Biochemistry 1988; 27: 6197-202.

[17] Brown DA, Rose JK. Sorting of GPI-anchored proteins to glycolipid-enriched membrane subdomains during transport to the apical cell surface. Cell 1992; 68: 533-44.

[18] Simons K, Toomre D. Lipid rafts and signal transduction. Nat Rev Mol Cell Biol 2000; 1: 31-9.

[19] Pike LJ. Lipid rafts: bringing order to chaos. J Lipid Res 2003; 44: 655-67.

[20] Anderson RG, Jacobson K. A role for lipid shells in targeting proteins to caveolae, rafts, and other lipid domains. Science 2002; 296: $1821-5$

[21] Pralle A, Keller P, Florin EL, Simons K, Horber JK. Sphingolipidcholesterol rafts diffuse as small entities in the plasma membrane of mammalian cells. J Cell Biol 2000; 148: 997-08.

[22] Varma R, Mayor S. GPI-anchored proteins are organized in submicron domains at the cell surface. Nature 1998; 394: 798-801.
[23] Schutz GJ, Kada G, Pastushenko VP, Schindler H. Properties of lipid microdomains in a muscle cell membrane visualized by single molecule microscopy. EMBO J 2000; 19: 892-901.

[24] Eggeling C, Ringemann C, Medda R, et al. Direct observation of the nanoscale dynamics of membrane lipids in a living cell. Nature 2009; 457: 1159-62.

[25] Mukherjee S, Maxfield FR. Membrane domains. Annu Rev Cell Dev Biol 2004; 20: 839-66.

[26] Lipowsky R. Budding of membranes induced by intramembrane domains. J de Phys II 1992; 2: 1825-40.

[27] Simons K, Vaz WL. Model systems, lipid rafts, and cell membranes. Annu Rev Biophys Biomol Struct 2004; 33: 269-95.

[28] van Meer G, Voelker DR, Feigenson GW. Membrane lipids: where they are and how they behave. Nat Rev Mol Cell Biol 2008; 9: 11224.

[29] Gaus K, Chklovskaia E, Fazekas de St GB, Jessup W, Harder T. Condensation of the plasma membrane at the site of $\mathrm{T}$ lymphocyte activation. J Cell Biol 2005; 171: 121-31.

[30] Gaus K, Le Lay S, Balasubramanian N, Schwartz MA. Integrinmediated adhesion regulates membrane order. J Cell Biol 2006; 174: 725-34.

[31] Feingold KR. The importance of lipids in cutaneous function. J Lipid Res 2007; 48: 2529-30.

[32] Menon GK, Feingold KR, Mao-Qiang M, Schaude M, Elias PM. Structural basis for the barrier abnormality following inhibition of HMG CoA reductase in murine epidermis. J Invest Dermatol 1992; 98: 209-19.

[33] Gniadecki R, Bang B. Flotillas of lipid rafts in transit amplifying cell-like keratinocytes. J Invest Dermatol 2003; 121: 522-8.

[34] Gniadecki R, Jemec GB. Lipid raft-enriched stem cell-like keratinocytes in the epidermis, hair follicles and sinus tracts in hidradenitis suppurativa. Exp Dermatol 2004; 13: 361-3.

[35] Watt FM. Role of integrins in regulating epidermal adhesion, growth and differentiation. EMBO J 2002; 21:3919-26.

[36] Gniadecki R, Christoffersen N, Wulf HC. Cholesterol-rich plasma membrane domains (lipid rafts) in keratinocytes: importance in the baseline and UVA-induced generation of reactive oxygen species. J Invest Dermatol 2002; 118: 582-8.

[37] Bang B, Gniadecki R, Gajkowska B. Disruption of lipid rafts causes apoptotic cell death in HaCaT keratinocytes. Exp Dermatol 2005; 14: 266-72.

[38] Vind-Kezunovic D, Wojewodzka U, Gniadecki R. Focal junctions retard lateral movement and disrupt fluid phase connectivity in the plasma membrane. Biochem Biophys Res Commun 2008; 365: 1-7.

[39] Lambert S, Vind-Kezunovic D, Karvinen S, Gniadecki R. Ligandindependent activation of the EGFR by lipid raft disruption. J Invest Dermatol 2006; 126: 954-62.

[40] Braccia A, Villani M, Immerdal L, et al. Microvillar membrane microdomains exist at physiological temperature. Role of galectin4 as lipid raft stabilizer revealed by "superrafts". J Biol Chem 2003 278: $15679-84$

[41] Viola A, Schroeder S, Sakakibara Y, Lanzavecchia A. T lymphocyte costimulation mediated by reorganization of membrane microdomains. Science 1999; 283: 680-2.

[42] Vind-Kezunovic D, Nielsen CH, Wojewodzka U, Gniadecki R. Line tension at lipid phase boundaries regulates formation of membrane vesicles in living cells. Biochim Biophys Acta 2008; 1778: $2480-6$.

This is an open access article licensed under the terms of the Creative Commons Attribution Non-Commercial License (http://creativecommons.org/licenses/by$\mathrm{nc} / 3.0 /$ ) which permits unrestricted, non-commercial use, distribution and reproduction in any medium, provided the work is properly cited. 